\title{
Effects of ejaculation on serum prostate-specific antigen levels
}

\author{
Kadir Demir ${ }^{1}$, Fatih Tarhan ${ }^{1}$, Asuman Orçun², Hasan Aslan¹, Akif Türk ${ }^{3}$
}

'Department of Urology, Dr. Lütfi Kırdar Kartal Training and Research Hospital,

İstanbul, Turkey

${ }^{2}$ Biochemistry Laboratory, Dr. Lütfi Kırdar Kartal Training and Research Hospital, İstanbul, Turkey

${ }^{3}$ Department of Urology, State Hospital, Akşehir, Konya,

Turkey

Submitted:

26.04.2013

Accepted:

09.09.2013

\section{Correspondence:}

Kadir Demir

Department of Urology, Dr.

Lütfi Kırdar Kartal Training

and Research Hospital, 34890 İstanbul, Turkey

Phone: +90 21644139 00-1840

E-mail: kadirde@gmail.com

(C) Copyright 2014 by Turkish Association of Urology

Available online at www.turkishjournalofurology.com

\begin{abstract}
Objective: The aim of this study is to evaluate the effect of ejaculation on serum total, free and complex prostate-specific antigen (PSA) levels and their effect on biopsy decisions.

Material and methods: Our study includes 47 men older than 45 years with symptomatic benign prostatic hyperplasia (BPH). Patients' PSA levels were under $2.5 \mathrm{ng} / \mathrm{mL}$, and there were no known conditions to preclude ejaculation. Eight patients with similar demographic, and clinical characteristics were chosen as a control group. With three days of sexual abstinence, blood samples were drawn for the measurement of baseline PSA levels, and then the patients were told to ejaculate. One, 5, 24 and 72 hours after ejaculation, serum total, free and complexed PSA levels were measured. Serum PSA sampling was performed at the same intervals in the control group without ejaculation.
\end{abstract}

Results: The mean age of the patients was $59.13 \pm 1.38$ years in the study group, and $63.75 \pm 3.13$ years in the control group. The mean international prostate symptom scores (IPSS) were $11.78 \pm 1.12$ and $11.63 \pm 3.32$ in the study, and the control groups, respectively. The mean baseline total, free, and complexed PSA values were $1.07 \pm 0.09,0.31 \pm 0.03$, and $0.71 \pm 0.08 \mathrm{ng} / \mathrm{mL}$, respectively. In the study group, total PSA value was found to be $1.29 \pm 0.12 \mathrm{ng} / \mathrm{mL}(\mathrm{p}=0.008)$ at first hour after ejaculation, and this change was statistically significant when compared with baseline values. Mean total PSA level rose up to $1.28 \pm 0.13 \mathrm{ng} / \mathrm{mL}(\mathrm{p}=0.05)$ by the $5^{\text {th }}$ hour after ejaculation, but this change was not statistically significant relative to the baseline values. Mean free PSA level rose after the first hour postejaculation to $0.37 \pm 0.04 \mathrm{ng} / \mathrm{mL}$ ( $\mathrm{p}=0.01)$ and after the fifth hour postejaculation to $0.37 \pm 0.04 \mathrm{ng} / \mathrm{mL}(\mathrm{p}=0.002)$, and these changes were statistically significant relative to the baseline values. There were no statistically significant changes at the other sampled times as for the total, free or complexed PSA serum levels. When all three values were considered individually, in only $2.12 \%$ of the patients, biopsy decisions could be affected by the elevation in PSA levels.

Conclusion: When the PSA value is borderline with respect to biopsy decisions, the effect of ejaculation on serum PSA levels may be clinically important. In these patients, ejaculation must be questioned, and repeated. PSA levels should be evaluated 24 hours after ejaculation. There were no statistically significant changes in the levels of complex PSA values. Further studies are needed to clarify the relationship between complexed PSA and ejaculation.

Key words: Ejaculation; prostate; PSA.

\section{Introduction}

Although prostate cancer is the most frequently encountered type of cancer in men, it ranks sixth among cancer-related deaths. ${ }^{[1]}$ After 1980s, with increasing number of transurethral resections, incidence of prostate cancer also has started to rise. Main increase occurred with the introduction of prostate-specific antigen (PSA) in urology practice in 1986. ${ }^{[2]}$ Introduction of PSA into current use has resulted in a serious increase in the recognition of localized, and early stage prostate cancer.

Prostate-specific antigen belongs to the family of human kallikrein gene. It is released into the seminal fluid in higher concentrations, and its conjugated (complex form), and free forms are found in the circulation. Under normal conditions, it is found in serum at lower concentrations. In the serum conjugated or complexed PSA is bound to alpha -3- macroglobuline, and alpha-1-antichymotrypsin. Macroglobulinbound PSA can not be detected with most of the available tests. ${ }^{[3-6]}$ Since PSA is not cancer -specific, but an organ-specific tumou marker, PSA values might differ in healthy individuals dependent on the age, ethnicity, and prostate volume, and in many benign disease states, and prostatic interventions as seen in malignant prostatic diseases. Controversial publications concerning the impact of ejaculation on PSA 
are available. ${ }^{[7]}$ Even if lower threshold values such as $2.6 \mathrm{ng} /$ $\mathrm{mL}$ were designated for the criteria of malignancy, prostate cancer was not detected in $75-80 \%$ of the biopsized patients. To refrain from unnecessary biopsies, and increase cancer-specificity of PSA, various PSA derivatives, and auxillary parameters were defined. In the prostate cancer, production of PSA does not increase. The factor which induces increases in PSA levels, is deterioration of the architecture of the prostate gland caused by prostate cancer. Increased rates of PSA might be released into serum together with disruption of the basal layer, and baseline membrane caused by prostate cancer. ${ }^{[8]}$

In the present study, in subjects with normal PSA levels, and without any indication of biopsy, postejaculatory changes in PSA levels which might exceed threshold values, and impact of these changes on the decisions of biopsy were investigated.

\section{Material and methods}

Sexually active males over 45 years of age who consulted to the urology outpatient clinic with medically untreated lower urinary tract symptoms (LUTS), and detected PSA levels of $<2.5 \mathrm{ng} /$ $\mathrm{dL}$ were included in the study. Patients carrying a suspicion of prostate cancer for any reason (digital rectal examination which raises the suspicion of prostate cancer, and doubtful metastatic lesions etc.), and those with conditions which prevent ejaculation (patients with transurethral catheters, urethral stenosis, and other obstructive causes) were excluded from the study. For the study, approval of the Ethics Committee was obtained from Dr. Lütfi Kırdar Kartal Training and Research Hospital with the decree \# 6, dated 05.15.2012. Written informed consent was received from all patients participating in the study.

All patients were interrogated using International Prostate Symptom Scoring System (IPSS), and subjected to complete urinalysis, digital rectal examination, serum total, free, and complexed PSA, and residual urine measurements, and uroflowmetric examinations. Besides, prostate volumes were calculated by means of suprapubically applied ultrasound transducer. Total (tPSA), free (fPSA) (Architect, Abbott), and complex PSA (cPSA) (ADVIA Centaur, Siemens) levels were measured using chemiluminescent microparticle immunoassay (CMIA) methods. From a total of 47 patients which comprise the study group, blood samples were drawn after a 3-day sexual abstinence period at baseline before ejaculation (PSA0), and at 1 (PSA1 hr), 5. (PSA5 hrs), 24. (PSA 24hrs), and 72. (PSA72 hrs) hours after ejaculation. In the control group blood samples were also drawn at aforementioned 5 time points for PSA measurements. All analyses were performed by the same urologist. The difference between the first, and second analyses was accepted as $\Delta$ value, $(\%) \Delta=(\Delta$ value/the first measurement value).

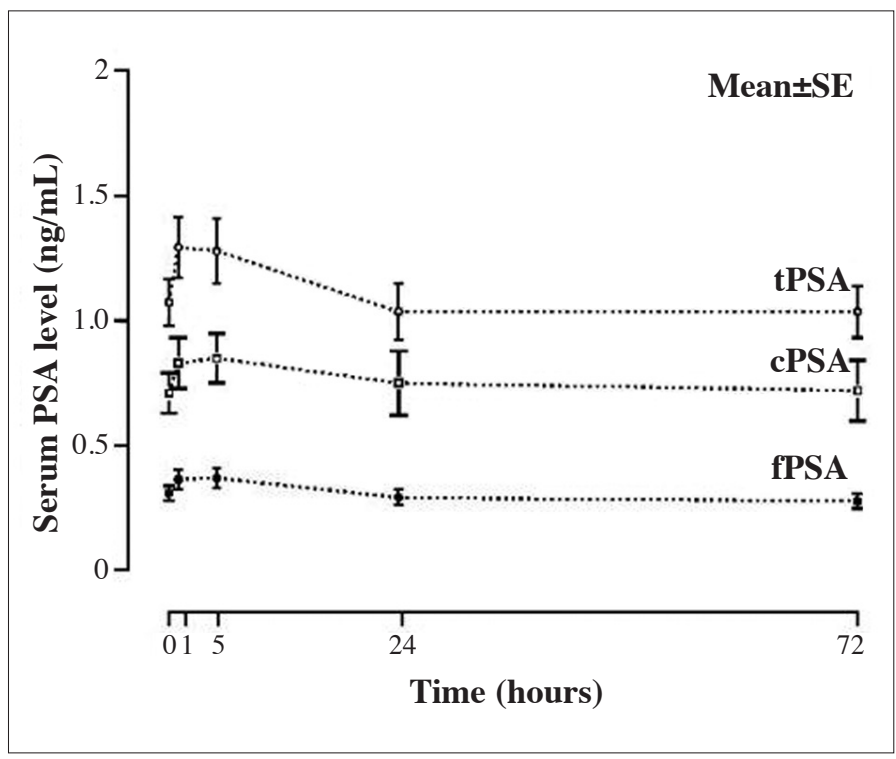

Figure 1. Preejaculatory baseline, total, free, and complexed PSA measurements, and postejaculatory changes from baseline as estimated at various time points

SE: standard error; tPSA: total prostate-specific antigen; cPSA: complexed prostate-specific antigen; fPSA: free prostate-specific antigen

\section{Statistical Analysis}

Data were presented as mean \pm standard error. Data were evaluated statistically using GraphPad Prism 5.0 Statistical Program, and 'paired - $t$-test, and Wilcoxon signed rank test, A p value of $<0.05$ was accepted as statistically significant.

\section{Results}

Mean age, IPSS, prostate volume, post-mictional residual urine volume, and Q max values in the study, and the control groups are given in Table 1.

Prostate-specific antigen values of the study, and the control groups are presented in Table 2, and Figure 1. In the study group baseline (tPSA0) and postejaculatory 1 . hour tPSA (tPSA1 hr) values were compared, and TPSA1 hr values were found to be statistically significantly higher $(\mathrm{p}=0.008)$, However tPSA5hr, and tPSA $1 \mathrm{hr}$ values were maintained at comparable levels which were not statistically significantly higher than baseline (tPSA0) values ( $\mathrm{p}=0.05)$. Pre-ejaculatory mean tPSA24 hr, and tPSA72 hr levels dropped to baseline total PSA levels after ejaculation without any significant difference between baseline values ( $\mathrm{p}=0.86$, and $\mathrm{p}=0.63$, respectively) (Table 2 , Figure 1 ).

In the study group, postejaculatory $1^{\text {st }}(\mathrm{p}=0.01)$, and $5^{\text {th }}$ hour fPSA ( $\mathrm{p}=0.002$ ) values were statistically significantly higher than corresponding baseline fPSA values However postejaculatory 24 ., and 72 . hour values did not demonstrate statistically 
significant differences from baseline fPSA values $(\mathrm{p}=0.72$, and $\mathrm{p}=0.06$, respectively).

In the study group, comparison between baseline, and postejaculatory 1, 5., 24., and 72. hour- cPSA values could not reveal any statistically significant difference between values estimated at different time points $(\mathrm{p}>0.05)$.

In the control group any statistically significant difference was not found between total, and free PSA values estimated at baseline, and at 1., 5., 24., and 72.-hours without prior ejaculation $(\mathrm{p}>0.05)$.

Postejaculatory serum tPSA values decreased in one $(1 / 47$; $2.1 \%)$, and increased in $46(46 / 47 ; 97.9 \%)$ patients when compared with the baseline values. Mean difference between postejaculatory, and baseline tPSA levels was $0.15 \pm 0.06 \mathrm{ng} / \mathrm{mL}$ (0.12-2.59) which corresponded to $14.80 \pm 3.55 \%(6.7-147.1 \%)$ change from the baseline.

Postejaculatory serum tPSA, cPSA, and fPSA levels increased above threshold values in $2.1 \%(1 / 47), 2.1 \%$ (1/47), and $1.2 \%$ (1/47) of the patients, respectively.

\section{Discussion}

In the early diagnosis of prostate cancer, PSA per se is thought to be the best diagnostic criterion, however its limited specificity rises concerns about its utility. One of the attempts at

\begin{tabular}{|c|c|c|}
\hline & Study $(n=47)$ & Control $(n=8)$ \\
\hline Age (year) & $59.13 \pm 1.38$ & $63.75 \pm 3.13$ \\
\hline IPSS & $11.78 \pm 1.12$ & $11.63 \pm 3.32$ \\
\hline Prostate volume (mL) & $43.18 \pm 1.91$ & $36.50 \pm 2.83$ \\
\hline Residual urine volume $(\mathrm{mL})$ & $19.14 \pm 3.60$ & $32.50 \pm 32.50$ \\
\hline $\mathrm{Q}_{\max }(\mathrm{mL} / \mathrm{sec})$ & $16.66 \pm 0.71$ & $14.00 \pm 2.19$ \\
\hline
\end{tabular}

increasing its specificity is to disclose conditions which might induce increases in PSA levels apart from prostate cancer, and rule out these conditions during evaluation process. ${ }^{[9]}$

As mentioned before, the effects of various therapeutic, diagnostic procedures, and clinical conditions on serum PSA levels were reported previously. Ejaculation has been claimed to be one of the factors which might increase PSA values, and its effect has been evaluated in some studies. ${ }^{[10-16]}$ Previous studies have been performed largely with total PSA levels, and free PSA levels at a lesser extent. We haven't encounter any literature study investigating variations in postejaculatory complexed PSA levels when compared with baseline values. Summary of previous studies is shown in Table 3.

As seen in Table 3, actually most of the studies performed couldn't detect any change in PSA levels when compared with baseline values. Increased PSA levels were detected in two studies, while they were lower than baseline values in one case. Only two cases were performed in comparison with the control group. In these studies postejaculatory PSA measurement times, and methods differed widely. Our study differs from other investigations in that it was also performed with complexed PSA. Time interval between ejaculation, and PSA sampling was standardized. The presence of a control group is an advantage of our study, however lack of a risk group for prostate cancer, its inability to represent the patient population seen in daily practice, and scarce number of patients in the control group constitute limitations of our study.

Yavaşçaoğulları et al. ${ }^{[10]}$ investigated changes in PSA values at 24. hours, and 5. days after ejaculation in 25 patients aged 23-25 years, and detected mean decrease of $22.37 \%$ in PSA levels in 16 patients, and mean increase of $38.3 \%$ in 6 patients which were not statistically significant when compared with the control group of 20 patients.

McAleer et al. ${ }^{[12]}$ indicated that the time passed from the act of ejaculation, and blood sampling varying between 1 , and 24 hours (mean time interval, $14.6 \mathrm{hrs}$ ) had no impact on serum

Table 2. PSA values (ng\mL) detected at different time points in the study, and control groups

\begin{tabular}{|c|c|c|c|c|c|c|}
\hline & \multicolumn{3}{|c|}{ Study group* } & \multicolumn{3}{|c|}{ Control group ${ }^{\#}$} \\
\hline Baseline & $1.07 \pm 0.09$ & $0.31 \pm 0.03$ & $0.71 \pm 0.08$ & $1.77 \pm 0.71$ & $0.51 \pm 0.04$ & $1.37 \pm 0.64$ \\
\hline 1.hr & $1.29 \pm 0.12$ & $0.37 \pm 0.04$ & $0.83 \pm 0.10$ & $1.75 \pm 0.72$ & $0.50 \pm 0.07$ & $1.29 \pm 0.57$ \\
\hline 5. hr & $1.28 \pm 0.13$ & $0.37 \pm 0.04$ & $0.85 \pm 0.10$ & $1.74 \pm 0.68$ & $0.50 \pm 0.03$ & $1.28 \pm 0.53$ \\
\hline 24. hr & $1.04 \pm 0.11$ & $0.29 \pm 0.03$ & $0.75 \pm 0.13$ & $1.75 \pm 0.76$ & $0.49 \pm 0.09$ & $1.30 \pm 0.59$ \\
\hline
\end{tabular}


Table 3. Representative studies which investigated effects of ejaculation on serum PSA levels

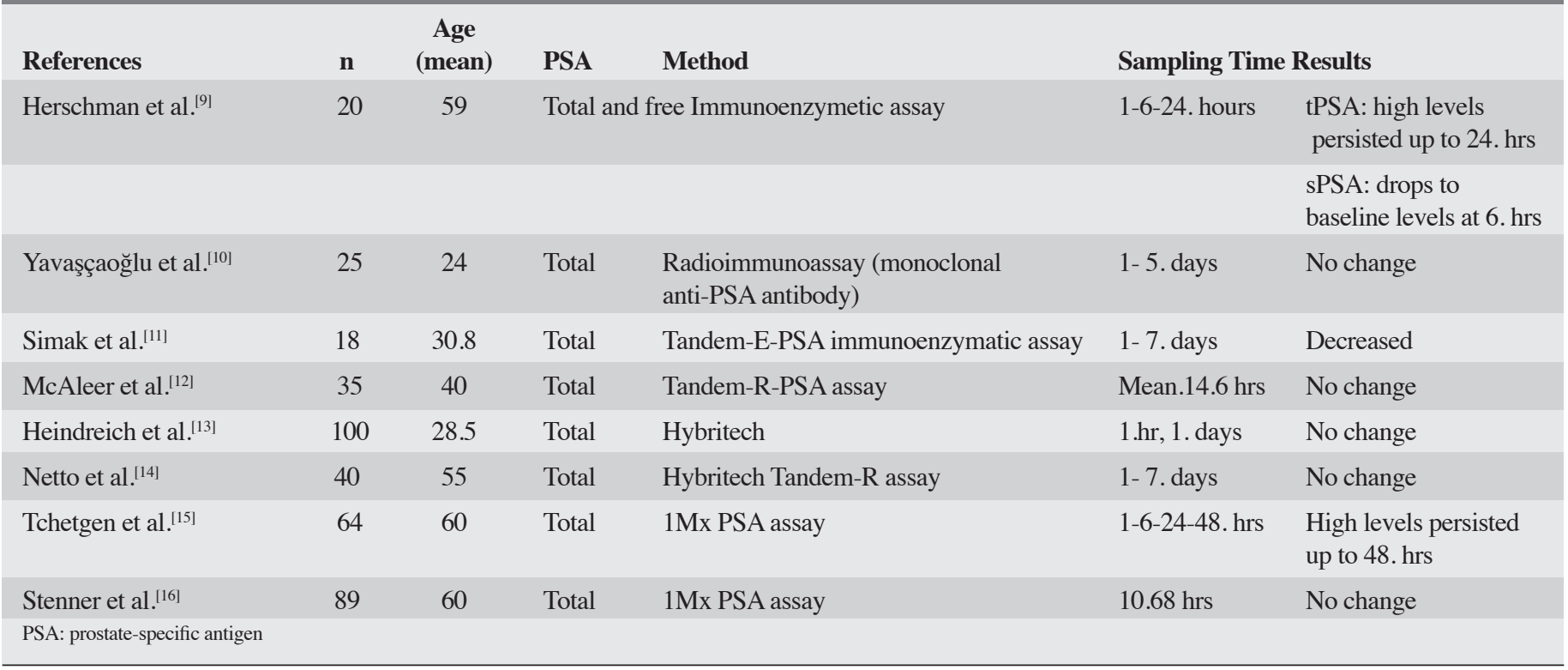

PSA levels. However time of ejaculation within 24 hours was determined by interrogating with the patient, and potential masking of early changes by late-term changes was overlooked. Besides, the state of sexual abstinence required for healthy evaluation of baseline PSA was not indicated. Similarly, Netto et al. ${ }^{[14]}$ reported that in patients within the age bracket which constitute risk for prostate cancer, and those with benign prostatic hyperplasia, PSA measurements were performed at postejaculatory 1., and 7. days. The authors detected higher mean PSA values at postejaculatory 1. days which did not represent a statistically significant change from the baseline.

Heindeinreich et al. ${ }^{[13]}$ evaluated PSA levels in young patients with a mean age of 28.5 years, and mean baseline PSA, postejaculatory 1., and 24. hour-PSA levels as $0.85 \mathrm{ng} / \mathrm{mL}, 0.95$ $\mathrm{ng} / \mathrm{mL}$, and $0.91 \mathrm{ng} / \mathrm{mL}$, respectively without any statistically significant change from baseline values. The authors indicated that in young men ejaculation had no effect on serum PSA levels, and also emphasized that lack of any risk group for prostate cancer in their study population was a limitation of their study.

Stenner et al. ${ }^{[16]}$ indicated statistically insignificant postejaculatory changes in PSA in 89 patients aged 60 years, however these patients were evaluated within postejaculatory 48 hours. (mean, 10.64 $\pm 7.9 \mathrm{hrs}$ ). When PSA $>4 \mathrm{ng} / \mathrm{mL}$ was taken as a cut-off value, they reported that postejaculatory changes in only $6 \%$ of their patients might alter their decision of performing biopsy. The time interval from the onset of ejaculation, and measurement of PSA was not standardized which might conceal potentially early rises in PSA levels. Besides, the authors indicated that increases in PSA levels might occur especially in patients with higher baseline PSA values. They also noted that ejaculation might be an important factor in the utilization of PSA particularly for clinical staging (as a prognostic factor in the prediction of nodal involvement, capsular invasion or systemic disease).

Herschman et al. ${ }^{[9]}$ analyzed changes in total, and free PSA levels at postejaculatory 1, 6, and 24 . hours in 20 volunteers with an average age of 59 years, and found statistically significantly higher mean total PSA concentrations when compared with baseline values estimated at abovementioned time points. In the same study, mean free PSA values at baseline $(0.3 \mathrm{ng} / \mathrm{mL})$, and postejaculatory 1. (0.5 ng/mL), 6. (0.3 ng/mL), and 24. (0.3 ng/ $\mathrm{mL}$ ) hours were determined, and only levels of free PSA estiamted at postejaculatory 1 . hours differed statistically significantly from baseline values. In their study, the authors especially emphasized that when $4 \mathrm{ng} / \mathrm{mL}$ was accepted as a threshold value of PSA, postejaculatory changes in PSA would not alter their decision of performing biopsy, and also drew attention to their smaller scale patient population. They accepted threshold value of PSA, and percentage PSA for the indication iof biopsy as $2.5 \mathrm{ng} / \mathrm{mL}$, and $<20 \%$, respectively. The authors also reported that baseline PSA values of their $3(3 / 18 ; 17 \%)$ patients did not meet criteria of biopsy, while their postejaculatory 1 . hour values increased and complied with these criteria, and higher PSA levels were maintained for 24 hours in one of them.

Tchetgen et al. ${ }^{[15]}$ detected statistically significantly higher levels of total PSA at postejaculatory 1., 6., and 24. hours in patients aged $\geq 50$ years. These higher postejaculatory PSA levels were detected in $87 \%$ of their patients, and PSA values dropped to baseline levels in $92 \%$ of the patients at 24 . hours. 
Authors reported peak PSA levels especially at postejaculatory 1. hours. PSA values dropped to baseline levels after relatively longer postejaculatory periods in the elderly, and those with higher baseline values. In their study any threshold value was not determined for baseline PSA.

Simak et al ${ }^{[11]}$ included young patients who had been investigated for the causes of their infertility in their study, and reported a postejaculatory drop in their PSA levels, The authors explained this phenomenon with postejaculatory decrease in PSA pool and resultant decrease in the amount of intravascular PSA leakage.

Any literature study has not investigated postejaculaatory changes in complexed PSA levels. In our study, we couldn't find any statistically significant changes in complexed PSA levels relative to baseline values. Therefore, we can say that postejaculatory increases in PSA levels might be rather related to increase in free PSA values.

Free PSA combines with ACT to form complexed PSA in a hitherto debatable anatomic site. Higher amounts of free PSA, rather than complexed PSA might be released or after release of free PSA, complexed PSA might be formed in the serum. In vitro studies have supported the assumption that process of complex formation between free PSA, and ACT lasts longer than the duration of complex formation between fPSA,and $\alpha-2$ macroglobulin. ${ }^{[17]}$ This stability of complexed PSA might explain lack of any significant postejaculatory changes in complexed PSA. In support of the abovementioned assertions, Tarhan et al. ${ }^{[17]}$ reported very high levels of total, and free PSA following prostatic massage, contrary to small variations in complexed PSA concentrations.

Serum PSA levels are dependent on the production of PSA, and its passage into circulation. Normal prostate columnar epithelium constitutes PSA -secreting acinar tissue, and PSA is transported through ejaculatory ducts. During its transportation, PSA enters into systemic circulation. These cells are separated from lymphatics, and capillaries by a baseline membrane, and cellular layer. Compression of these tissues by hyperplastic tissues might explain the behaviour of PSA (higher PSA values) in these patients. ${ }^{[14]}$

Another hypothesis which explains higher PSA levels in the elderly asserts that physiologic barrier can weaken, and become more permeable resulting in passage of higher amounts of PSA into blood circulation. Ductal obstruction, acinar dilatation, retention of secretions due to additional impact of benign prostatic hyperplasia together with weakened baseline membrane, and increased permeability of the barrier might increase PSA leakage during ejaculation. ${ }^{[15]}$ Besides, contractions of the pelvic muscle, and periprostatic tissues facilitate passage of PSA into circulation. ${ }^{[16]}$
When we consider previous studies performed in conjunction with these explained basic mechanisms, decrease in PSA values observed generally in studies performed with young patients can be associated with evacuation of PSA pool after ejaculation in the presence of an intact barrier, but in the absence of the impact of compression of hyperplastic tissues, and ductal obstruction which restrict passage of PSA into circulation. In well-designed studies performed with symptomatic BPH or elderly patients, observance of higher PSA levels at various frequencies, and for diverse time periods supports the mechanisms which implicate compression of the hyperplastic tissue, and disrupted barrier function of the baseline membrane for the variations in PSA values.

Study limitations, lack of any risk group for prostate cancer in the study which represent the patient population seen in the daily practice, and scarce number of the patients in the control group constitute limitations of our study.

We have detected that tPSA, and PPSA values in our study increased immediately after ejaculation, and dropped to baseline values at postejaculatory 24. hours. Besides, in only one patient all PSA values elevated over threshold levels. Especially, in patients with baseline PSA values closer to cutoff values which might be an indication for biopsy, ejaculation soon before PSA measurements might influence the decision of performing biopsy. Since, ejaculation within 24 hours might increase PSA levels in some patients, before PSA measurements one-day sexual abstinence might be recommended. Although complexed PSA results are statistically insignificant, same concern exists for complexed psa in patients with closer values to cut-off levels. In addition, clinically cPSA is not superior over other PSA forms. Conduction of further studies is needed to reveal the association between the ejaculation, and complexed PSA.

Ethics Committee Approval: Ethics committee approval was received for this study from the ethics committee of Dr. Lütfi Kırdar Training and Research Hospital.

Informed Consent: Written informed consent was obtained from patients who participated in this study.

Peer-review: Externally peer-reviewed.

Author Contributions: Concept - F.T., K.D.; Design - K.D., F.T.; Supervision - F.T., A.T.; Funding - K.D., H.A.; Materials - A.O., K.D.; Data Collection and/or Processing - K.D., A.O., H.A., A.T.; Analysis and/or Interpretation - F.T., K.D.; Literature Review - K.D., F.T., A.T.; Writer - K.D., F.T.; Critical Review - F.T., H.A., A.T., A.O.; Other K.D., A.T., H.A.

Conflict of Interest: No conflict of interest was declared by the authors. 
Financial Disclosure: The authors declared that this study has received no financial support.

\section{References}

1. Jemal A, Bray F, Center MM, Ferlay J, Ward E, Forman D. Global cancer statistics. CA Cancer. J Clin 2011;61:69-90.

2. Parkin DM, Bray F, Ferlay J, Pisani P. Global cancer statistics, 2002. CA Cancer J Clin 2005;55:74-108.

3. Stenman UH, Leinonen J, Alfthan H, Rannikko S, Tuhkanen K, Alfthan O. A complex between prostate specific antigen and alantichymotrypsin is the major form of prostate-specific antigen in serum of patients with prostatic cancer: assay of the complex improves clinical sensitivity for cancer. Cancer Res 1991;51:222-6.

4. Lilja H, Christensson A, Dahlh U, Matikainen MT, Nilsson O, Pettersson K, et al. Prostate-specific antigen in serum occurs predominantly in complex with alpha l-antichymotrypsin. Clin Chem 1991;37:1618-25.

5. McCormack RT, Rittenhouse HG, Finlay JA, Sokoloff RL, Wang TJ, Wolfert RL, et al. Molecular forms of prostate-specific antigen and the human kallikrein gene family: a new era. Urology 1995;45:729-44.

6. Diamandis EP, Yu H. Nonprostatic sources of prostatespecific antigen. Urol Clin N Amer 1997;24:275-82.

7. Loeb S, Carter HB. Early Detection, Diagnosis, and Staging of Prostate Cancer. Campbell-Walsh Urology: Wein AJ, Kavoussi LR, Novick AC, Partin AW, Peters CA (eds.) Saunders-Elsevier, Philadelphia, Chapter 99 2011:2763-70.

8. Getzenberg RH, Partin AW. Prostate Cancer Tumor Markers Campbell-Walsh Urology: Wein AJ, Kavoussi LR, Novick AC,
Partin AW, Peters CA (eds.) Saunders-Elsevier, Philadelphia, Chapter 98 2012:2748-62.

9. Herschman JD, Smith DS, Catalona WJ. Effect of ejaculation on serum total and free prostate-specific antigen concentrations. Urology 1997;50:239-43.

10. Yavaşçaoglu I, Savci V, Oktay B, Simşek U, Ozyurt M. The effects of ejaculation on serum prostate-specific antigen (PSA). Int Urol Nephrol 1998;30:53-8.

11. Simak R, Madersbacher S, Zhang ZF, Maier U. The impact of ejaculation on serum prostate specific antigen. J Urol 1993;150:895-7.

12. McAleer JK, Gerson LW, McMahon D, Geller L. Effect of digital rectal examination (and ejaculation) on serum prostate-specific antigen after twenty-four hours. A randomized, prospective study. Urology 1993;41:111-2.

13. Heidenreich A, Vorreuther R, Neubauer S, Westphal J, Engelmann UH, Moul JW. The influence of ejaculation on serum levels of prostate specific antigen. J Urol 1997;157:209-11.

14. Netto NR Jr, Apuzzo F, de Andrade E, Srulzon GB, Cortado PL, Lima ML. The effects of ejaculation on serum prostate specific antigen. J Urol 1996;155:1329-31.

15. Tchetgen MB, Song JT, Strawderman M, Jacobsen SJ, Oesterling JE. Ejaculation increases the serum prostate-specific antigen concentration. Urology 1996;47:511-6.

16. Stenner J, Holthaus K, Mackenzie SH, Crawford ED. The effect of ejaculation on prostate-specific antigen in a prostate cancerscreening population. Urology 1998;51:455-9.

17. Tarhan F, Orçun A, Küçükercan İ, Çamursoy N, Kuyumcuoğlu U. Effect of prostatic massage on serum complexed prostate-specific antigen levels. Urology 2005;66:1234-8. 